\title{
Higgs precision physics: the next step
}

\author{
Giampiero Passarino*t \\ Dipartimento di Fisica Teorica, Università di Torino, Italy \\ INFN, Sezione di Torino, Italy \\ E-mail: giampiero@to.infn.it
}

Higgs precision physics is examined from the point of view of systematizing the notion of theoretical uncertainty. A proposal is made for a formal definition of theoretical uncertainty expressed in terms of a nuisance (continuous) parameter that encodes estimates on missing higher orders in perturbation theory.

11th International Symposium on Radiative Corrections (Applications of Quantum Field Theory to Phenomenology)

22-27 September 2013

Lumley Castle Hotel, Durham, UK

\footnotetext{
* Speaker.

${ }^{\dagger}$ Work supported by MIUR under contract 2001023713_006 and by Compagnia di San Paolo under contract ORTO11TPXK.
} 


\section{Introduction}

After the discovery of a resonance at $123-126 \mathrm{GeV}$ at LHC [1,2] we are entering a new phase, prove or disprove that it is the Standard Model (SM) Higgs boson. High precision becomes an essential ingredient; indeed, any machine that can measure couplings with limited precision can only claim a discovery of a SM-like Higgs boson. If the same machine can measure couplings that differ significantly from the predicted SM values, then it is possible to rule out the SM Higgs boson at that machine.

From the point of view of Higgs precision physics there are several vital steps or stages one must climb. Here we have selected theoretical precision and missing higher orders, other most important issues include inadequacy of on-shell Higgs physics, see Refs. [3, 4, 5, 6] and also Refs. [7, 8] and Higgs effective Lagrangians, see Ref. [9] and the references therein (see also Ref. [10]).

\section{Theoretical accuracy}

The traditional way for estimating theoretical uncertainties associated to collider physics is based on the notion of QCD (factorization and renormalization) scale variation [11, 12]. However, it is well known that there are several examples in the literature where the QCD scale uncertainty of the $n$th order overestimates the $(n+1)$ th order. Higher-order calculations for Higgs boson production confirm that the gg-channel is the dominant one (70\%) and that there are large $K$ factors, see Ref. [13]. To summarize, the perturbative series for $g g$-fusion converges slowly and scale variations underestimate the next order (only $\mu_{\mathrm{R}}$-dependence is significant here). $\mathrm{N}^{3} \mathrm{LO}$ computation in gluon channel is underway, see Ref. $[14,15]$, while approximate $\mathrm{N}^{3} \mathrm{LO}$ results have been already obtained in Ref. [16].

There is also an open and debatable question on how to assign a probability distribution function (pdf) to the MHOU and the generally accepted one is based on a Gaussian (or log-normal) distribution; what to use for the standard deviation, remains an open problem. Alternatively, it can be assumed that the pdf is a flat-box representing a credible interval (or Bayesian confidence interval). In this context it is worth noting the original proposal made in Ref. [12] which is based on the introduction of a flat (uninformative) Bayesian prior.

Recently we have introduced [17] the concept of MHO(MHOU), missing higher order (uncertainty), which has to do with the truncation error in the perturbative expansion and a proposal has been made for predicting higher orders of the perturbative expansion using the well-known concept of series acceleration, i.e. one of a collection of sequence transforms ( $\mathrm{T}$ ) for improving the rate of convergence of a series. If the original series is divergent, the transform acts as an extrapolation method; in the case of infinite sums, they have the effect that sums that formally diverge may return a result that can be interpreted as evaluation of analytic extension of the series for the sum. Given partial sums

$$
S=\sum_{k=0}^{\infty} \gamma_{k} z^{k}, \quad S_{n}=\sum_{k=0}^{n} \gamma_{k} z^{k}
$$

the whole strategy is based on the fact that one can predict the coefficients with $k>n$ by constructing an approximant with the known terms of the series $\left(\gamma_{0}, \ldots, \gamma_{n}\right)$ and expanding the approximant 
in a Taylor series. The first $n$ terms of this series will exactly agree with those of the original series and the subsequent terms may be treated as the predicted coefficients. Therefore, if $S_{1}, \ldots, S_{n}$ are known, one computes

$$
T_{n}-S_{n}=\bar{\gamma}_{n+1} z^{n+1}+\mathscr{O}\left(z^{n+2}\right)
$$

and $\bar{\gamma}_{n+1}$ is the prediction for $\gamma_{n+1}$. Here, $T_{n}$ is a transformed sequence, e.g. one of the Levin's $\tau$ transforms (see Refs. [18, 19, 20, 21]), Wynn's epsilon algorithm [22], Brezinski's $J$-algorithm [23] or Weniger's $\delta$-transform [19]. We also know that all transforms basically differ in the choice of the remainder estimates. A good choice should satisfy the following asymptotic condition [24]

$$
R_{n}=\frac{S-S_{n}}{\omega_{n}} \sim c, \quad n \rightarrow \infty
$$

where $\omega_{n}$ is the remainder. For instance, Levin selects $\omega_{n}=\Delta S_{n-1}$, where $\Delta$ is the usual forwarddifference operator, $\Delta S_{n}=S_{n+1}-S_{n}$

The main application developed in Ref. [17] concerns the process $\mathrm{gg} \rightarrow \mathrm{H}$ where one writes the cross-section in terms of the lowest-order (LO) and of a $K$-factor admitting a formal power expansion in $\alpha_{\mathrm{s}}\left(\mu_{\mathrm{R}}\right)$ (i.e. $1+\sum_{n=1}^{\infty} \alpha_{\mathrm{s}}^{n}\left(\mu_{\mathrm{R}}\right) K_{\mathrm{gg}}^{n}$ ). The first two (non trivial) coefficients of the expansion are known (11.879 and 72.254) whereas, for the third one, we have an approximate calculation available (with its own intrinsic uncertainty), see Ref. [16].

The conclusion of Ref. [17] is that, to a very good accuracy, the "true" cross-section value is bracketed by the estimations of Eq.(2.4), with all other transforms falling very close to the right boundary.

$$
\begin{gathered}
\sigma_{\mathrm{gg}} \in\left[\sigma_{\mathrm{gg}}^{\mathrm{S}, 3 \mid 3}, \sigma_{\mathrm{gg}}^{\delta, 6 \mid 3}\right] \\
\sigma_{\mathrm{gg}}^{\mathrm{S}, \mathrm{N} \mid n}=\sigma_{\mathrm{gg}}^{0}\left(\mu=M_{\mathrm{H}}\right) S_{\mathrm{N} \mid n}\left(\mu=M_{\mathrm{H}}\right) \quad \text { and } \quad \sigma_{\mathrm{gg}}^{\delta, \mathrm{N} \mid n}=\sigma_{\mathrm{gg}}^{0}\left(\mu=M_{\mathrm{H}}\right) \delta_{\mathrm{N}, n}\left(\mu=M_{\mathrm{H}}\right)
\end{gathered}
$$

where we have introduced the $N$ th partial sum with $n$ known coefficients

$$
S_{\mathrm{N}, n}=\sum_{k=0}^{n} \alpha_{\mathrm{s}}^{k}\left(\mu_{\mathrm{R}}\right) K_{\mathrm{gg}}^{k}+\sum_{k=n+1}^{\mathrm{N}} \alpha_{\mathrm{s}}^{k}\left(\mu_{\mathrm{R}}\right) \bar{K}_{\mathrm{gg}}^{k}
$$

and the corresponding Weniger-transform [19] of order $N$ based on $n$ known partial sums, see Ref. [17] for details. To summarize, the estimate of Ref. [17] tells us that the "true" cross-section value is between the last known calculation and the largest prediction obtained by spanning over $\mathscr{T}$, the whole (discrete) set of STs, i.e. $\max _{T \in \mathscr{T}} \sigma_{\mathrm{gg}}^{T, \mathrm{~N} \mid 3}$ (in this case $T$ is the Weniger transform of order $N=6$ ). The resulting uncertainty, taken as a flat interval (uninformative prior), corresponds to a $16.37 \%$ and its intrinsic uncertainty, induced by the error on the third coefficient, brings us to $26.01 \%$.

One should mention in this regard that there is no proof of the uniqueness of the result reconstructed from its asymptotic series. There is only numerical evidence that all sequence transforms produce a result within a small interval, which allows us to assign an uninformative prior, in the Bayesian sense.

In conclusion, going from NNLO to $\mathrm{N}^{3} \mathrm{LO}[16]$ produces an increase of $\approx 17 \%$ on the crosssection; our completion gives an additional $+7 \%$. The corresponding pdf could be derived by 

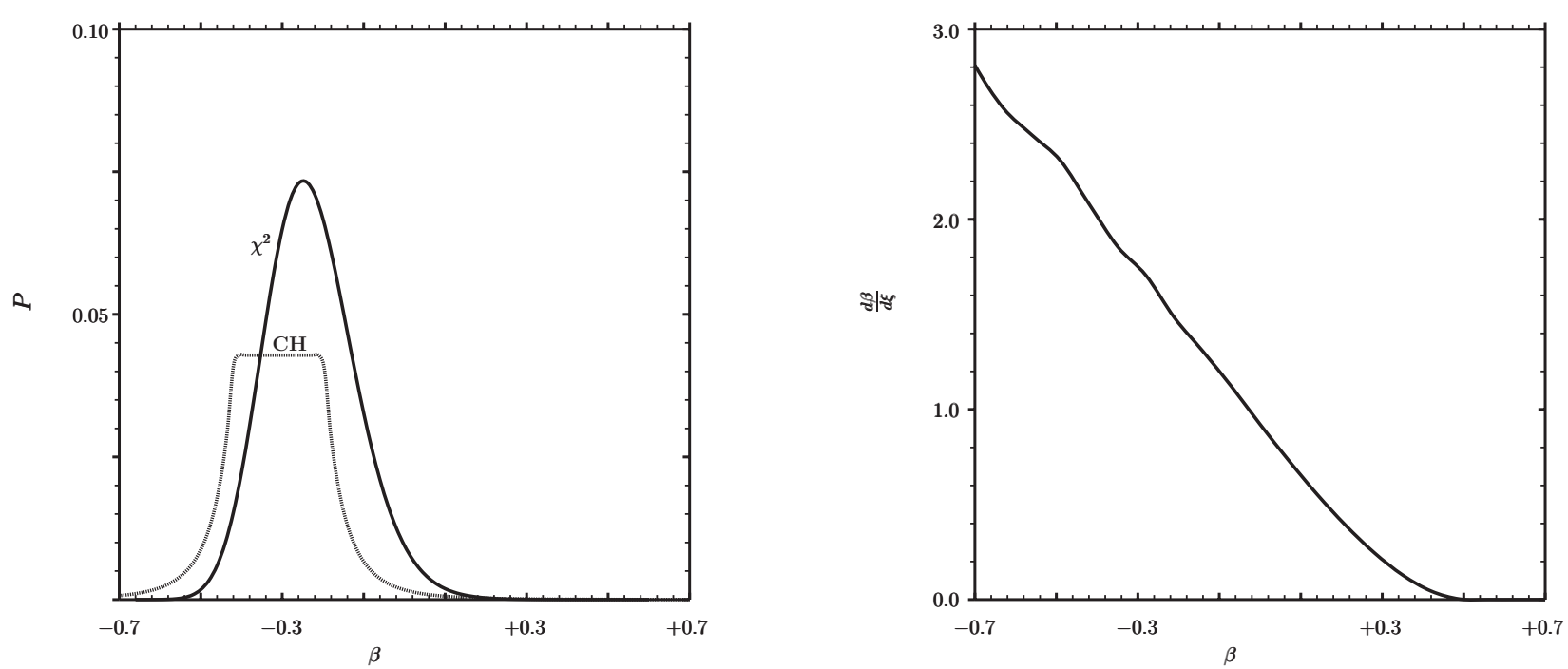

Figure 1: In the left panel we show $P_{\chi^{2}}$ defined in Eq.(2.11) and $P_{\mathrm{CH}}$ defined in Eq.(2.15). In the right panel we show the $d \beta / d \eta$, defined in Eq.(2.14)

following the work of Ref. [12]: the prior, however, requires scanning over $\mathscr{T}$, the discrete (finite) set of different approximants.

Therefore, the new proposal is to parametrize the effect of MHO by using a single, continuous, variable for which we consider the parameter entering shifted sequence transformations. For instance, a Levin $\tau$-transform can be further generalized by introducing a shift parameter $\beta$ : consider a series where the coefficients $\gamma_{1}, \ldots, \gamma_{3}$ are known; we can use $\gamma_{1,2}$, tune $\beta$ to have $\bar{\gamma}_{3}=\gamma_{3}$ and use the same value of $\beta$, to predict $\gamma_{4}$ etc.

The original motivation for introducing the shift parameter was the following: a Levin-like transform of order $k$ requires $k+1$ terms of the original series and the first $k+1$ terms of the series expansion of the transform coincide with those of the original series for any value of $\beta$. Tuning $\beta$ one can predict the $k+3$ th term with a much higher accuracy, as discussed in Ref. [25].

Our perspective is slightly different: we propose to use $\beta$ as the parameter effectively describing MHO effects. To this end, we introduce

$$
\begin{gathered}
\tau_{k}^{n}(\beta)=\frac{\sum_{i=i_{0}}^{k} W^{\tau}(n, k, i, \beta) S_{n+i}}{\sum_{i=i_{0}}^{k} W^{\tau}(n, k, i, \beta)} \\
W^{\tau}(n, k, i, \beta)=(-1)^{i}\left(\begin{array}{c}
k \\
i
\end{array}\right) \frac{(\beta+n+i)_{k-1}}{\Delta S_{n+i-1}}
\end{gathered}
$$

and we write $\tau_{k}$ for $\tau_{k}^{0}$; furthermore, $\tau_{k \mid l}$ denotes the approximant $\tau$ where only the first $l$ partial sums are exact.

At the moment there is a residual uncertainty on the value of $K_{\mathrm{gg}}^{3}$, from Ref. [16] we find (at $\sqrt{s}=8 \mathrm{TeV}$ and $\left.\mu_{\mathrm{R}}=\mu_{\mathrm{F}}=M_{\mathrm{H}}\right)$

$$
\alpha_{\mathrm{s}}^{3}\left[K_{\mathrm{gg}}^{3 \mathrm{c}} \pm \Delta K_{\mathrm{gg}}^{3}\right]=0.527 \pm 0.043
$$


Using $K_{\mathrm{gg}}^{1,2}$ we predict

$$
\bar{K}_{\mathrm{gg}}^{3}(\beta)=2 \frac{\beta+1}{\beta+2} \frac{\left(K_{\mathrm{gg}}^{2}\right)^{2}}{K_{\mathrm{gg}}^{1}}
$$

and construct a distribution

$$
\chi^{2}=\left(\frac{\bar{K}_{\mathrm{gg}}^{3}(\beta)-K_{\mathrm{gg}}^{3 \mathrm{c}}}{2 \Delta K_{\mathrm{gg}}^{3}}\right)^{2}
$$

Introducing $y=\chi^{2} / 2$ and $z^{2}=y$ we obtain $P_{\chi^{2}}(\beta)$, which is the pdf for the initial uncertainty on the third coefficient of the series,

$$
P_{\chi^{2}}(\beta)=\frac{2}{\sqrt{\pi}} e^{-z^{2}} \frac{d z}{d \beta}
$$

Coming back to the original problem we define $\sigma_{\mathrm{gg}}(\beta)$ as

$$
\sigma_{\mathrm{gg}}(\beta)=\sigma_{\mathrm{gg}}^{0}\left(\mu=M_{\mathrm{H}}\right) \tau_{6 \mid 2}(\beta)
$$

where we are using only the first two coefficients of the series and derive $\beta_{ \pm}$as the solutions of the equations

$$
\sigma_{\mathrm{gg}}\left(\beta_{-}\right)=\sigma_{\mathrm{gg}}^{\mathrm{S}, 3 \mid 3}, \quad \sigma_{\mathrm{gg}}\left(\beta_{+}\right)=\sigma_{\mathrm{gg}}^{\delta, 6 \mid 3}
$$

It can be seen that $\sigma_{\mathrm{gg}}(\beta)$ is a monotonically increasing function of $\beta$. Therefore, $\sigma_{\mathrm{gg}}(\beta)$ interpolates continuously between the two extremes of the interval of Eq.(2.4) and the MHOU is parametrized in terms of a single function of $\beta$. We can define the corresponding (MHOU) pdf by following again the work of Ref. [12]; however, the natural variable that should enter the formal uninformative prior is

$$
\eta=\frac{\sigma_{\mathrm{gg}}(\beta)}{\sigma_{\mathrm{gg}}^{\mathrm{S}, 3 \mid 3}}-1
$$

insofar as such quantity measures deviations of the "true" cross-section from the known perturbative approximation and our prior is uninformative w.r.t. $\eta$. Therefore, we consider

$$
P_{\chi^{2}}(\eta)=P_{\chi^{2}}(\beta) \frac{d \beta}{d \eta}, \quad P_{\mathrm{CH}}(\eta)=P_{\mathrm{CH}}(\beta) \frac{d \beta}{d \eta}
$$

introduce $\eta_{+}=\eta\left(\beta_{+}\right)$and

$$
P_{\mathrm{CH}}(\eta)=\frac{2}{3 \Delta \eta}\left\{\begin{array}{lll}
\left(\frac{\Delta \eta}{\eta_{+}-\eta}\right)^{5} \text { if } & \eta<1 \\
1 & \text { if } & 1<\eta<\eta_{+} \\
\left(\frac{\Delta \eta}{\eta-1}\right)^{5} & \text { if } & \eta>\eta_{+}
\end{array}\right.
$$

with $\Delta \eta=\eta_{+}-1$. Obviously, $P_{\mathrm{CH}}(\beta)=P_{\mathrm{CH}}(\eta) d \eta / d \beta$. Finally, $P_{\chi^{2}}$ and $P_{\mathrm{CH}}$ can be combined by taking a convolution in $\eta$-space, $q(\eta)=P_{\chi^{2}} \otimes P_{\mathrm{CH}}$. Starting from this definition, we can define a more general reference prior: let $\mathbf{x}=\left(x_{1}, \ldots, x_{n}\right)(\mathbf{x} \in X)$ be the entire observation vector for 
the process and $p(\{x\}, \eta)$ the density describing the model, including parton distribution functions (PDF).

The advantage of this method relies on a formal definition of theoretical uncertainty in terms of a nuisance (continuous) parameter $\eta$. Therefore, we have a data vector $\mathbf{x}$ with probability density $p, \mathbf{x}$ are the parameter of interest and $\eta$ is the nuisance. The information to be expected (reference prior) is [26]

$$
I=\int_{X} d^{n} x \int_{-\infty}^{+\infty} d \eta p(\{x\}, \eta) q(\eta) \ln \frac{p(\{x\}, \eta)}{q(\eta)}
$$

Maybe reference prior could not be considered as the "best" choice of a uninformative prior but can be considered as the most successful one. Although there is no guarantee that the frequentistmatching property holds (e.g. when one uses a "naive" ("flat") uninformative prior such as the uniform distribution or a Gaussian distribution with a huge variance), a posterior 95\%-credibility interval is also (at least, approximately) a $95 \%$-confidence interval in the frequentist sense. Therefore, one can also construct confidence intervals in the presence of the new nuisance parameter (representing theoretical uncertainty) by using standard methods [27].

\section{Conclusions}

Using gluon fusion as a case study, we know that QCD scale dependence gives at most a lower bound on the theoretical uncertainty. Following the proposal made in Ref. [17], where the first (known) orders are used to construct an all-order approximant and the difference all-order fixed-order is used to estimate theoretical uncertainty, we give a formal definition of theoretical uncertainty in terms of a nuisance (continuous) parameter and construct the corresponding (posterior) probability distribution function. 


\section{References}

[1] ATLAS Collaboration, G. Aad et al., Observation of a new particle in the search for the Standard Model Higgs boson with the ATLAS detector at the LHC, Phys. Lett. B 716 (2012) 1-29, arXiv:1207.7214 [hep-ex].

[2] CMS Collaboration, S. Chatrchyan et al., Observation of a new boson at a mass of $125 \mathrm{GeV}$ with the CMS experiment at the LHC, Phys. Lett. B 716 (2012) 30-61, arXiv:1207. 7235 [hep-ex ] .

[3] N. Kauer and G. Passarino, Inadequacy of zero-width approximation for a light Higgs boson signal, JHEP 1208 (2012) 116, arXiv: 1206.4803 [hep-ph] .

[4] G. Passarino, Higgs Boson Production and Decay: Dalitz Sector, arXiv: 1308.0422 [hep-ph].

[5] G. Passarino, Higgs Interference Effects in $\mathrm{gg} \rightarrow \mathrm{ZZ}$ and their Uncertainty, JHEP 1208 (2012) 146, arXiv:1206.3824 [hep-ph].

[6] S. Goria, G. Passarino, and D. Rosco, The Higgs Boson Lineshape, Nucl.Phys. B864 (2012) 530-579, arXiv:1112.5517 [hep-ph].

[7] M. Bonvini, F. Caola, S. Forte, K. Melnikov, and G. Ridolfi, Signal-background interference effects for $\mathrm{gg} \rightarrow \mathrm{H} \rightarrow \mathrm{W}^{+} \mathrm{W}^{-}$beyond leading order, Phys.Rev. D88 (2013) 034032, arXiv: 1304.3053 [hep-ph].

[8] F. Caola and K. Melnikov, Constraining the Higgs boson width with ZZ production at the LHC, Phys.Rev. D88 (2013) 054024, arXiv: 1307.4935 [hep-ph] .

[9] LHC Higgs Cross Section Working Group Collaboration, S. Heinemeyer et al., Handbook of LHC Higgs Cross Sections: 3. Higgs Properties, arXiv:1307.1347 [hep-ph].

[10] G. Passarino, NLO Inspired Effective Lagrangians for Higgs Physics, Nucl.Phys. $\mathbf{8 8 6 8}$ (2013) 416-458, arXiv:1209.5538 [hep-ph].

[11] LHC Higgs Cross Section Working Group Collaboration, S. Dittmaier et al., Handbook of LHC Higgs Cross Sections: 1. Inclusive Observables, arXiv:1101.0593 [hep-ph].

[12] M. Cacciari and N. Houdeau, Meaningful characterisation of perturbative theoretical uncertainties, JHEP 1109 (2011) 039, arXiv: 1105.5152 [ hep-ph] .

[13] R. Boughezal, F. Caola, K. Melnikov, F. Petriello, and M. Schulze, Higgs boson production in association with a jet at next-to-next-to-leading order in perturbative QCD, JHEP 1306 (2013) 072, arXiv:1302.6216 [hep-ph].

[14] C. Anastasiou, C. Duhr, F. Dulat, and B. Mistlberger, Soft triple-real radiation for Higgs production at N3LO, JHEP 1307 (2013) 003, arXiv: 1302 . 4379 [hep-ph] .

[15] S. Buehler and A. Lazopoulos, Scale dependence and collinear subtraction terms for Higgs production in gluon fusion at N3LO, arXiv:1306.2223 [hep-ph] .

[16] R. D. Ball, M. Bonvini, S. Forte, S. Marzani, and G. Ridolfi, Higgs production in gluon fusion beyond NNLO, arXiv:1303.3590 [hep-ph].

[17] A. David and G. Passarino, How well can we guess theoretical uncertainties?, Phys.Lett. B726 (2013) 266-272, arXiv:1307.1843 [hep-ph].

[18] D. Levin, , Int. J. Comput. Math. 3 (1973) 371.

[19] E. J. Weniger, , Comput. Phys. Rep. 10 (1989) 189-371. 
[20] E. J. Weniger, , J. Math. Phys. 45 (2004) 1209.

[21] A. Sidi, , J. Comput. Appl. Math. 37 (1980) 7.

[22] P. Wynn, , Math. Tab. Aids Comput. 10 (1956) 91.

[23] C. Brezinski, , Numer. Math. 35 (1980) 175.

[24] E. J. Weniger, Nonlinear sequence transformations: Computational tools for the acceleration of convergence and the summation of divergent series, arXiv:math/0107080 [math-ca] .

[25] D. Roy and R. Bhattacharya, Prediction of unknown terms of a sequence and its application to some physical problems, Annals Phys. 321 (2006) 1483-1523.

[26] J. Berger, J. Bernardo, and D. Sun, The formal definition of reference priors, Ann. Statist. 37(2) (2009) 905-938.

[27] B. Sen, M. Walker, and M. Woodroofe, On the unified method with nuisance parameters, Statistica Sinica 19 (2009) 301-314. 